\title{
EXPLICIT UPPER BOUNDS FOR THE SPECTRAL DISTANCE OF TWO TRACE CLASS OPERATORS
}

\author{
OSCAR F. BANDTLOW AND AYŞE GÜVEN
}

\begin{abstract}
Given two trace class operators $A$ and $B$ on a separable Hilbert space we provide an upper bound for the Hausdorff distance of their spectra involving only the distance of $A$ and $B$ in operator norm and the singular values of $A$ and $B$. By specifying particular asymptotics of the singular values our bound reproduces or improves existing bounds for the spectral distance. The proof is based on lower and upper bounds for determinants of trace class operators of independent interest.
\end{abstract}

\section{INTRODUCTION}

Given an arbitrary compact operator $A$ on a separable Hilbert space an interesting question of practical importance is to determine its spectrum $\sigma(A)$. One way of tackling it numerically is to reduce the infinite-dimensional problem to a finite-dimensional one by manufacturing a sequence of finite rank operators $\left(A_{k}\right)_{k=1}^{\infty}$ converging to $A$ in operator norm and using the fact that the eigenvalues of $A_{k}$, converge to the eigenvalues of $A$. In practice, one has to stop after a finite number of steps. If there is interest in error estimates, the problem arises how to explicitly bound the distance of the spectrum of a finite rank approximant $A_{k}$ to the spectrum of $A$ in a suitable sense. A popular choice is the Hausdorff metric, the definition of which we briefly recall.

For $z \in \mathbb{C}$ and a compact subset $\sigma \subset \mathbb{C}$ let

$$
d(z, \sigma)=\inf _{\lambda \in \sigma}|z-\lambda|
$$

denote the distance of $z$ to $\sigma$. Given two compact subsets $\sigma_{1}, \sigma_{2} \subset \mathbb{C}$ their Hausdorff distance is given by

$$
\operatorname{Hdist}\left(\sigma_{1}, \sigma_{2}\right)=\max \left\{\hat{d}\left(\sigma_{1}, \sigma_{2}\right), \hat{d}\left(\sigma_{2}, \sigma_{1}\right)\right\}
$$

where

$$
\hat{d}\left(\sigma_{1}, \sigma_{2}\right)=\sup _{\lambda \in \sigma_{1}} d\left(\lambda, \sigma_{2}\right) .
$$

It is not difficult to see that the Hausdorff distance is a metric on the set of compact subsets of $\mathbb{C}$.

The finite-dimensional prototype of an explicitly computable bound for the Hausdorff distance of the spectra of two $n \times n$ matrices $A$ and $B$, also known as the spectral distance of $A$ an $B$ in this context, is due to Elsner [7, sharpening various

Date: September 27, 2014.

1991 Mathematics Subject Classification. 47A55, 47B10, 4710.

Key words and phrases. Spectral distance, spectral variation, trace class operators, determinants. 
earlier bounds (see [5, Chapter VIII] for a discussion) beginning with Ostrowski [16], and reads as follows

$$
\operatorname{Hdist}(\sigma(A), \sigma(B)) \leq(\|A\|+\|B\|)^{1-\frac{1}{n}}\|A-B\|^{\frac{1}{n}},
$$

where $\|\cdot\|$ denotes the operator norm corresponding to the Euclidean norm on $\mathbb{C}^{n}$. Note that if the operator norm is taken with respect to any other (non-Euclidean) norm on $\mathbb{C}^{n}$, then (11) does not hold (see [14]).

Unfortunately, a straightforward extension of this formula to the infinite-dimensional setting by simply passing to the limit fails, due to the presence of the exponent $1 / n$. However, versions of (11) hold for $A$ and $B$ algebraic elements of a Banach algebra of degree less than $n$ (see [6]).

Infinite-dimensional versions of (10) have so far been obtained essentially by one method going back to Henrici 12 which relies on writing a given compact operator as a perturbation of a quasi-nilpotent operator by a normal operator with the same spectrum as the original operator. Using this approach upper bounds for the spectral distance of Schatten class operators have been obtained by Gil' in a series of papers (see [8] and references therein for an overview) and one of the authors (see [1, 2]). Similar bounds, less sharp, but valid more generally for operators in symmetrically normed ideals are due to Pokrzywa [17, using the same method. For recent extensions of the results in $[8$ to unbounded operators with inverses in the Schatten classes see [9, 10].

This article grew out of an attempt to bypass the Henrici argument and instead make Elsner's delightful determinant based proof of (1) work in the infinitedimensional setting. By considering determinants and keeping track of the singular values of the operators involved we arrive at a version of Elsner's formula (1) which holds for trace class operators and which yields new bounds even in finite dimensions.

It is organised as follows. In Section 2 we provide some background on trace class operators $A$ and the corresponding determinants $\operatorname{det}(I-A)$. In the following section (Section 3) we first derive a lower bound for $\operatorname{det}\left(I-z^{-1} A\right)$, where $A$ is trace class, expressible solely in terms of the distance of $z$ to the spectrum of $A$ and the singular values of $A$. This bound is of independent interest and does not seem to have appeared in the literature yet. Next, for $z$ an eigenvalue of a bounded operator $B$, we derive an upper bound for $\operatorname{det}\left(I-z^{-1} A\right)$ in terms of the distance of $z$ to the spectrum of $A$ and $\|A-B\|$. In Section 4 we combine the upper and lower bounds to obtain our main result. We finish (Section 5) by comparing our bound to Elsner's and show that it reduces to or improves the bounds in [1, 2].

\section{TRACE CLASS OPERATORS AND THEIR DETERMINANTS}

In this section we briefly recapitulate some facts about trace class operators and their determinants, which will be crucial for what is to follow.

Let $H$ be a separable Hilbert space with scalar product $(\cdot, \cdot)$. We write $L(H)$ for the Banach space of bounded linear operators on $H$ equipped with the uniform operator norm $\|\cdot\|$. For $A \in L(H)$ the spectrum and the set of eigenvalues will be denoted by $\sigma(A)$ and $\sigma_{p}(A)$, respectively, while the resolvent set will be denoted by $\rho(A)$.

If $A \in L(H)$ is a compact operator, we use $\left(\lambda_{n}(A)\right)_{n \in \mathbb{N}}$ to denote its eigenvalue sequence, counting algebraic multiplicities and ordered by decreasing modulus so 
that

$$
\left|\lambda_{1}(A)\right| \geq\left|\lambda_{2}(A)\right| \geq \cdots
$$

If $A$ has only finitely many non-zero eigenvalues, we set $\lambda_{n}(A)=0$ for $n>N$, where $N$ denotes the number of non-zero eigenvalues of $A$. For $n \in \mathbb{N}$, the $n$-th singular value of $A$ is given by

$$
s_{n}(A)=\sqrt{\lambda_{n}\left(A^{*} A\right)} \quad(n \in \mathbb{N}),
$$

where $A^{*}$ denotes the Hilbert space adjoint of $A$.

Eigenvalues and singular values satisfy a number of inequalities known as Weyl's inequalities. The most important of them is the multiplicative Weyl inequality (see, for example, [11, Chapter VI, Theorem 2.1])

$$
\prod_{k=1}^{n}\left|\lambda_{k}(A)\right| \leq \prod_{k=1}^{n} s_{k}(A) \quad(\forall n \in \mathbb{N}) .
$$

The following additive version follows from the multiplicative one (see, for example, [11, Chapter VI, Corollary 2.3])

$$
\sum_{k=1}^{n}\left|\lambda_{k}(A)\right| \leq \sum_{k=1}^{n} s_{j}(A) \quad(\forall n \in \mathbb{N})
$$

and so does the following (see, for example, [11, Chapter VI, Corollary 2.5])

$$
\prod_{k=1}^{n}\left(1+r\left|\lambda_{k}(A)\right|\right) \leq \prod_{k=1}^{n}\left(1+r s_{k}(A)\right) \quad(\forall r \geq 0, \forall n \in \mathbb{N}) .
$$

We now recall the notion of a trace class operator as an element of the set

$$
S_{1}(H)=\left\{A \in L(H): A \text { compact and }\|A\|_{1}=\sum_{k=1}^{\infty} s_{k}(A)<\infty\right\} .
$$

It turns out that $\|\cdot\|_{1}$ is a norm on $S_{1}(H)$ turning it into a Banach space (see, for example, [11, Chapter VI, Corollary 3.2 and Theorem 4.1]).

With any a trace class operator $A \in S_{1}(H)$ it is possible to associate a determinant given by

$$
\operatorname{det}(I-A)=\prod_{k=1}^{\infty}\left(1-\lambda_{k}(A)\right),
$$

which, in view of (4) and the summability of the singular values, is well-defined and satisfies the bound

$$
|\operatorname{det}(I-A)| \leq \prod_{k=1}^{\infty}\left(1+\left|\lambda_{k}(A)\right|\right) \leq \prod_{k=1}^{\infty}\left(1+s_{k}(A)\right) \leq \exp \left(\|A\|_{1}\right) .
$$

In particular, this implies that $z \mapsto \operatorname{det}(I-z A)$ is an entire function of exponential type zero (see, for example, [11, Chapter VII, Theorem 4.3]). 


\section{UPPER AND LOWER BOUNDS FOR DETERMINANTS}

In this section we shall derive both upper and lower bounds for the determinant of a trace class operator $A$ expressible in terms of the function $F_{A}:[0, \infty) \rightarrow[0, \infty)$ given by

$$
F_{A}(r)=\prod_{k=1}^{\infty}\left(1+r s_{k}(A)\right)
$$

which is well-defined, real-analytic and strictly monotonically increasing.

Before deriving these bounds we briefly recall two more facts about singular values. The first is a useful alternative characterisation in terms of approximation numbers (see, for example, [11, Chapter VI, Theorem 1.5])

$$
s_{n}(A)=\inf \{\|A-F\|: F \in L(H), \operatorname{rank}(F)<n\} \quad(\forall n \in \mathbb{N}) .
$$

Using this characterisation the second fact follows: if $G$ is a closed subspace of $H$ with orthogonal projection $P$ we have (see, for example, 11, Chapter VI, Corollary 1.4])

$$
s_{n}\left(\left.P A\right|_{P(H)}\right) \leq s_{n}(A) \quad(\forall n \in \mathbb{N}) .
$$

By definition of the determinant of a trace class oprator $A$, the function $z \mapsto$ $\operatorname{det}\left(I-z^{-1} A\right)$ has zeros at the non-zero eigenvalues of $A$. The following proposition provides a new lower bound for the behaviour of $z \mapsto \operatorname{det}\left(I-z^{-1} A\right)$ in the vicinity of a zero solely in terms of the function $F_{A}$ defined in (5) and the distance of $z$ to the spectrum of $A$.

Proposition 3.1. Let $A \in S_{1}(H)$. Then, for any $z \in \rho(A)$ with $z \neq 0$, we have

$$
\left|\operatorname{det}\left(I-z^{-1} A\right)\right|^{-1} \leq F_{A}\left(\frac{1}{d(z, \sigma(A))}\right) \text {. }
$$

Proof. For brevity, write $\lambda_{k}=\lambda_{k}(A)$ and $s_{k}=s_{k}(A)$. Then, for $z \in \rho(A)$ with $z \neq 0$, we have

$$
\begin{aligned}
& \left|\operatorname{det}\left(I-z^{-1} A\right)\right|^{-1}=\prod_{k=1}^{\infty}\left|\frac{1}{1-z^{-1} \lambda_{k}}\right|=\prod_{k=1}^{\infty}\left|1+\frac{\lambda_{k}}{z-\lambda_{k}}\right| \\
& \quad \leq \prod_{k=1}^{\infty}\left(1+\frac{\left|\lambda_{k}\right|}{\left|z-\lambda_{k}\right|}\right) \leq \prod_{k=1}^{\infty}\left(1+\frac{\left|\lambda_{k}\right|}{d(z, \sigma(A))}\right) \leq \prod_{k=1}^{\infty}\left(1+\frac{s_{k}}{d(z, \sigma(A))}\right),
\end{aligned}
$$

where the last inequality follows from (44).

We now provide an upper bound for $\operatorname{det}\left(I-z^{-1} A\right)$ whenever $z$ is an eigenvalue of an operator $B \in L(H)$ expressible in terms of the distance of $z$ to $\sigma(A)$ and the distance of $A$ and $B$.

Proposition 3.2. Suppose that $\operatorname{dim} H=\infty$ and that $A, B \in L(H)$. If $A$ has finite rank, then for any $z \in \rho(A) \cap \sigma_{p}(B)$ we have

$$
\left|\operatorname{det}\left(I-z^{-1} A\right)\right| \leq \frac{\|A-B\|}{d(z, \sigma(A))} \prod_{k=1}^{n}\left(1+\frac{s_{k}(A)}{d(z, \sigma(A))}\right)
$$

where $n=\operatorname{rank}(A)$. 
Proof. Fix $z \in \rho(A) \cap \sigma_{p}(B)$. Note that since $\operatorname{dim} H=\infty$, we have $0 \in \sigma(A)$, so $z \neq 0$. Let $e$ be an eigenvector of $B$ corresponding to $z$ and let $E$ denote the smallest closed linear span containing the range of $A$ and $e$. Clearly, $E$ is an invariant subspace of $A$ with $\nu:=\operatorname{dim} E \leq n+1$. Writing $T_{E}$ for the restriction of an operator $T \in L(H)$ to $E$, we have

$$
\lambda_{k}(A)= \begin{cases}\lambda_{k}\left(A_{E}\right) & \text { if } k \leq \nu, \\ 0 & \text { if } k>\nu .\end{cases}
$$

Thus

$$
\left|\operatorname{det}\left(I-z^{-1} A\right)\right|=\prod_{k=1}^{\nu}\left|\lambda_{k}\left(I_{E}-z^{-1} A_{E}\right)\right| \leq \prod_{k=1}^{\nu} s_{k}\left(I_{E}-z^{-1} A_{E}\right),
$$

where we have used the multiplicative Weyl inequality 1 (2).

Now, for $k<\nu$, we have the bound

$$
s_{k}\left(I_{E}-z^{-1} A_{E}\right) \leq 1+|z|^{-1} s_{k}\left(A_{E}\right) \leq 1+|z|^{-1} s_{k}(A),
$$

which follows from ([6). In order to treat the $\nu$-th factor define $K: E \rightarrow E$ by $K=I_{E}-z^{-1} P B_{E}$, where $P$ denotes the orthogonal projection onto $E$. Since $K e=0$ we have $\operatorname{rank} K<\nu$, so

$$
\begin{aligned}
& s_{\nu}\left(I_{E}-z^{-1} A\right) \leq\left\|\left(I_{E}-z^{-1} P A_{E}\right)-K\right\| \\
& \quad=|z|^{-1}\left\|P B_{E}-P A_{E}\right\| \leq|z|^{-1}\|A-B\|,
\end{aligned}
$$

where we have used (6) and (7). Combining (8), (9), and (10) and taking into account that $|z| \geq d(z, \sigma(A))$, since $0 \in \sigma(A)$, we have

$$
\left|\operatorname{det}\left(I-z^{-1} A\right)\right| \leq \frac{\|A-B\|}{d(z, \sigma(A))} \prod_{k=1}^{\nu-1}\left(1+\frac{s_{k}(A)}{d(z, \sigma(A))}\right),
$$

and the assertion follows.

Remark 3.3. Note that in the above proof we have only used the hypothesis $\operatorname{dim} H=\infty$ to conclude that $|z| \geq d(z, \sigma(A))$. Inspection of the proof shows that the following finite-dimensional analogue of the above result holds.

Let $A, B \in L\left(\mathbb{C}^{n}\right)$. Then for any $z \in \rho(A) \cap \sigma(B)$ with $z \neq 0$ we have

$$
\left|\operatorname{det}\left(I-z^{-1} A\right)\right| \leq \frac{\|A-B\|}{d(z, \sigma(A) \cup\{0\})} \prod_{k=1}^{n-1}\left(1+\frac{s_{k}(A)}{d(z, \sigma(A) \cup\{0\})}\right) .
$$

Here we have used the fact that both the eigenvector of $B$ and the range of $A$ trivially belong to the same $n$-dimensional space.

The above proposition can be extended to arbitrary trace class operators using a standard approximation procedure.

\footnotetext{
${ }^{1}$ In fact there is equality here, since for any $\nu \times \nu$ matrix $M$ we have

$$
\prod_{k=1}^{\nu}\left|\lambda_{k}(M)\right|^{2}=|\operatorname{det}(M)|^{2}=\operatorname{det}\left(M^{*} M\right)=\prod_{k=1}^{\nu} s_{k}(M)^{2} .
$$
}


Proposition 3.4. Let $H$ be infinite-dimensional and suppose that $A \in S_{1}(H)$ and $B \in L(H)$. Then for any $z \in \rho(A) \cap \sigma_{p}(B)$ we have

$$
\left|\operatorname{det}\left(I-z^{-1} A\right)\right| \leq \frac{\|A-B\|}{d(z, \sigma(A))} F_{A}\left(\frac{1}{d(z, \sigma(A))}\right) .
$$

Proof. Since $A$ is trace class it has a Schmidt representation of the form

$$
A x=\sum_{k=1}^{\infty} s_{k}(A)\left(x, a_{k}\right) b_{k} \quad(\forall x \in H),
$$

where $\left(a_{k}\right)_{k=1}^{\infty}$ and $\left(b_{k}\right)_{k=1}^{\infty}$ are orthonormal systems in $H$ (see [11, Chapter VI, Theorem 1.1]). For any $n \in \mathbb{N}$ we now define a finite-rank operator $A_{n}$ by

$$
A_{n} x=\sum_{k=1}^{n} s_{k}(A)\left(x, a_{k}\right) b_{k} \quad(\forall x \in H) .
$$

A short calculation shows that

$$
s_{k}\left(A_{n}\right)= \begin{cases}s_{k}(A) & \text { for } k \leq n, \\ 0 & \text { for } k>n\end{cases}
$$

and that

$$
\lim _{n \rightarrow \infty}\left\|A_{n}-A\right\|_{1}=0 .
$$

Now fix $z \in \rho(A) \cap \sigma_{p}(B)$. Since $z \in \rho(A)$ standard perturbation theory implies that there is $N \in \mathbb{N}$ such that $z \in \rho\left(A_{n}\right)$ for all $n \geq N$ (see, for example, [11, Chapter II, Theorem 4.1]). Thus, by Proposition 3.2, we have

$$
\left|\operatorname{det}\left(I-z^{-1} A_{n}\right)\right| \leq \frac{\left\|A_{n}-B\right\|}{d\left(z, \sigma\left(A_{n}\right)\right)} F_{A}\left(\frac{1}{d\left(z, \sigma\left(A_{n}\right)\right)}\right) \quad(\forall n \geq N),
$$

where we have used the fact that $F_{A_{n}}(r) \leq F_{A}(r)$ for every $r \in[0, \infty)$, which follows from (11).

In order to conclude the proof we make a number of observations. Since the determinant is Lipschitz-continuous on the unit ball of $S_{1}(H)$ (see, for example, [18, Theorem 6.5]) we have

$$
\lim _{n \rightarrow \infty} \operatorname{det}\left(I-z^{-1} A_{n}\right)=\operatorname{det}\left(I-z^{-1} A\right) .
$$

Furthermore, since the spectrum of $A$ is discrete, the spectrum of $A_{n}$ converges to the spectrum of $A$ in the Hausdorff metric (see [15, Theorem 3]), so

$$
\lim _{n \rightarrow \infty} d\left(z, \sigma\left(A_{n}\right)=d(z, \sigma(A)) .\right.
$$

Finally, we clearly have

$$
\lim _{n \rightarrow \infty}\left\|A_{n}-B\right\|=\|A-B\| .
$$

The assertion now follows by taking the limit on both sides of (12). 


\section{Bounds FOR THE SPECTRAL DistAnCE}

The upper and lower bounds for the determinants obtained in the previous section can be combined to produce quantitative upper bounds for the spectral distance of two trace class operators. Before we proceed we require some more notation.

Let $\mathcal{F}$ denote the set of all strictly monotonically increasing functions $F$ : $[0, \infty] \rightarrow[0, \infty)$ with $\lim _{r \rightarrow \infty} F(r)=\infty$, and let $\mathcal{F}_{0}$ denote the subset of those $F \in \mathcal{F}$ with $F(0)=0$. Both $\mathcal{F}$ and $\mathcal{F}_{0}$ are partially ordered by defining

$$
F_{1} \leq F_{2}: \Leftrightarrow F_{1}(r) \leq F_{2}(r) \quad(\forall r \geq 0) \text {. }
$$

On $\mathcal{F}$ we define an operation $H$, which will allow us to express our bounds in a succinct way:

$$
\begin{gathered}
H: \mathcal{F} \rightarrow \mathcal{F}_{0} \\
F \mapsto H_{F}
\end{gathered}
$$

where $H_{F}$ is given by

$$
H_{F}(r)=\frac{1}{\tilde{F}^{-1}\left(\frac{1}{r}\right)},
$$

and $\tilde{F}^{-1}$ is the inverse of $\tilde{F} \in \mathcal{F}_{0}$ defined by $\tilde{F}(r)=r F(r)^{2}$.

For later use, we note the following simple consequences of the definition of $H$, the proofs of which are left as an exercise.

Lemma 4.1. Let $F_{1}, F_{2} \in \mathcal{F}$ and, for $m>0$, let $M_{m} \in \mathcal{F}$ be given by $M_{m}(r)=m r$. Then the following holds.

(1) If $F_{1} \leq F_{2}$ then $H_{F_{1}} \leq H_{F_{2}}$.

(2) If $F_{1}=F_{2} \circ M_{m}$, then $H_{F_{1}}=M_{m} \circ H_{F_{2}} \circ M_{m}^{-1}$.

We are now able to state and prove our main results.

Theorem 4.2. Suppose that $\operatorname{dim} H=\infty$ and that $A \in S_{1}(H)$. Then

$$
\hat{d}\left(\sigma_{p}(B), \sigma(A)\right) \leq H_{F_{A}}(\|A-B\|) \quad(\forall B \in L(H)) .
$$

Proof. Fix $B \in L(H)$. We need to show that

$$
d(z, \sigma(A)) \leq H_{F_{A}}(\|A-B\|) \quad\left(\forall z \in \sigma_{p}(B)\right) .
$$

For $z \in \sigma(A)$ the inequality above is trivially satisfied, so we shall assume that $z \in \rho(A) \cap \sigma_{p}(B)$. Noting that $z \neq 0$ (since $\operatorname{dim} H=\infty$ ), Propositions 3.1 and 3.4 yield

$$
\frac{1}{F_{A}\left(\frac{1}{d(z, \sigma(A))}\right)} \leq\left|\operatorname{det}\left(I-z^{-1} A\right)\right| \leq \frac{\|A-B\|}{d(z, \sigma(A))} F_{A}\left(\frac{1}{d(z, \sigma(A))}\right) .
$$

Hence

$$
\frac{1}{\|A-B\|} \leq \tilde{F}_{A}\left(\frac{1}{d(z, \sigma(A))}\right)
$$

where $\tilde{F}_{A}(r)=r F_{A}(r)^{2}$, so

which implies

$$
\tilde{F}_{A}^{-1}\left(\frac{1}{\|A-B\|}\right) \leq \frac{1}{d(z, \sigma(A))},
$$

$$
d(z, \sigma(A)) \leq \frac{1}{\tilde{F}_{A}^{-1}\left(\frac{1}{\|A-B\|}\right)}=H_{F_{A}}(\|A-B\|)
$$


and the assertion follows.

An immediate consequence of the previous theorem is the following quantitative bound for the Hausdorff distance of the spectra of two trace class operators.

Theorem 4.3. Suppose that $\operatorname{dim} H=\infty$ and that $A, B \in S_{1}(H)$. Then

$$
\operatorname{Hdist}(\sigma(A), \sigma(B)) \leq H_{F_{A, B}}(\|A-B\|),
$$

where $F_{A, B} \in \mathcal{F}$ is given by

$$
F_{A, B}(r)=\max \left\{F_{A}(r), F_{B}(r)\right\} .
$$

Proof. Using Theorem 4.2 together with Lemma 4.1 and the fact that $B$ is trace class we have

$$
\hat{d}(\sigma(B), \sigma(A))=\hat{d}\left(\sigma_{p}(B), \sigma(A)\right) \leq H_{F_{A, B}}(\|A-B\|) .
$$

But by symmetry we also have

$$
\hat{d}(\sigma(A), \sigma(B)) \leq H_{F_{A, B}}(\|A-B\|),
$$

and the assertion follows.

Remark 4.4. The condition $\operatorname{dim} H=\infty$ is not essential in the previous two theorems. Inspection of their proofs together with Remark 3.3 yield the following finite-dimensional analogue of Theorem 4.2 and 4.3

Let $A, B \in L\left(\mathbb{C}^{n}\right)$. Then

$$
\hat{d}(\sigma(B), \sigma(A) \cup\{0\}) \leq H_{F_{A}}(\|A-B\|),
$$

and

$$
\operatorname{Hdist}(\sigma(A) \cup\{0\}, \sigma(B) \cup\{0\}) \leq H_{F_{A, B}}(\|A-B\|) \text {. }
$$

\section{COMPARISON With OTHER BOUNDS}

In order to apply the bounds obtained in the previous section some knowledge of the singular values of the operators involved is required. Lemma4.1 implies that any upper bound for the singular values translates into a bound for the spectral distance of two trace class operators. We shall briefly discuss a number of possibilities, which either reproduce or improve existing bounds.

We start with a bound involving only one free parameter, namely the trace norm. Given $A \in S_{1}(H)$, we have

$$
F_{A}(r)=\prod_{k=1}^{\infty}\left(1+r s_{k}(A)\right) \leq \exp \left(r\|A\|_{1}\right) .
$$

Theorem 4.3 and Lemma 4.1 immediately give the following result, reproducing the known bound from [1, Theorem 5.2] obtained by a different method.

Corollary 5.1. Let $\operatorname{dim}(H)=\infty$. Then, for any $A, B \in S_{1}(H)$ not both of them the zero operator, we have

$$
\operatorname{Hdist}(\sigma(A), \sigma(B)) \leq m H_{G^{S}}\left(\frac{\|A-B\|}{m}\right),
$$

where $G^{S}(r)=\exp (r)$ and $m=\max \left\{\|A\|_{1},\|B\|_{1}\right\}$.

Another possibility is to demand that the singular values decay at a stretched exponential rate. This leads to the notion of exponential classes introduced in [2]. 
Definition 5.2. Let $a>0$ and $\alpha>0$. Then

$$
E(a, \alpha ; H)=\left\{A \in S_{1}(H):|A|_{a, \alpha}:=\sup _{k \in \mathbb{N}} s_{k}(A) \exp \left(a k^{\alpha}\right)<\infty\right\},
$$

is called the exponential class of type $(a, \alpha)$. The number $|A|_{a, \alpha}$ is called the $(a, \alpha)$ gauge or simply gauge of $A$.

Naturally occurring operators belonging to these classes include integral operators with analytic kernels (see 13), composition operators with strictly contracting holomorphic symbols, or, more generally, transfer operators arising from real analytic expanding maps, which play an important role in smooth ergodic theory (see [4). Certain operators on spaces of harmonic functions also belong to this class (see [3]).

Specialising to operators belonging to exponential classes, Theorem 4.3 and Lemma 4.1 yield the following.

Corollary 5.3. Let $\operatorname{dim} H=\infty$. Then, for any $A, B \in E(a, \alpha ; H)$ not both of them the zero operator, we have

$$
\operatorname{Hdist}(\sigma(A), \sigma(B)) \leq m H_{G_{a, \alpha}^{E}}\left(\frac{\|A-B\|}{m}\right),
$$

where

$$
G_{a, \alpha}^{E}(r)=\prod_{k=1}^{\infty}\left(1+r \exp \left(-a n^{\alpha}\right)\right)
$$

and $m=\max \left\{|A|_{a, \alpha},|B|_{a, \alpha}\right\}$.

By [2, Proposition 3.1] we have

$$
\log G_{a, \alpha}^{E}(r) \sim a^{1 / \alpha} \frac{\alpha}{1+\alpha}(\log r)^{1+1 / \alpha} \quad \text { as } r \rightarrow \infty
$$

and a short calculation shows that

$$
\log H_{G_{a, \alpha}^{E}}(r) \sim-(2 a)^{1 /(1+\alpha)}\left(\frac{1+\alpha}{\alpha}\right)^{\alpha /(1+\alpha)}|\log r|^{\alpha /(1+\alpha)} \quad \text { as } r \downarrow 0,
$$

thus Corollary 5.3 improves the bound in [2, Theorem 4.2] obtained using a different method.

Finally, we turn to the most stringent condition for the decay of the singular values, which arises when all but finitely many of the singular values vanish, or, in other words, if the operators involved are finite rank.

Using the same argument as in the previous two cases we obtain a bound that only relies on knowledge of the first singular value, that is, the operator norm. For better comparison with Elsner's bound we formulate it for $n \times n$ matrices (see Remark 4.4).

Corollary 5.4. Let $A, B \in L\left(\mathbb{C}^{n}\right)$. Then

$$
\operatorname{Hdist}(\sigma(A) \cup\{0\}, \sigma(B) \cup\{0\}) \leq m H_{G^{F}}\left(\frac{\|A-B\|}{m}\right),
$$

where

$$
G^{F}(r)=(1+r)^{n}
$$

and $m=\max \{\|A\|,\|B\|\}$. 
Note that the bound in the above corollary is of a form very similar to Elsner's bound, which for $A, B \in L\left(\mathbb{C}^{n}\right)$ can be written

$$
\operatorname{Hdist}(\sigma(A), \sigma(B)) \leq m H\left(\frac{\|A-B\|}{m}\right),
$$

where $m=\|A\|+\|B\|$ and $H(r)=r^{1 / n}$. Clearly, the bound in Corollary 5.4 cannot compete with the Elsner bound (except possibly in special cases), since

$$
H_{G^{F}}(r) \sim r^{1 /(2 n+1)} \quad \text { as } r \downarrow 0 .
$$

However, in deriving the bound above we have only used the first singular value. If we have information about the first two singular values, the bound can be improved.

Corollary 5.5. Let $A, B \in L\left(\mathbb{C}^{n}\right)$. Then

$$
\operatorname{Hdist}(\sigma(A) \cup\{0\}, \sigma(B) \cup\{0\}) \leq H_{G_{s_{1}, s_{2}}^{F}}(\|A-B\|),
$$

where

$$
G_{s_{1}, s_{2}}^{F}(r)=\left(1+r s_{1}\right)\left(1+r s_{2}\right)^{n-1},
$$

with $s_{1}=\max \left\{s_{1}(A), s_{1}(B)\right\}$ and $s_{2}=\max \left\{s_{2}(A), s_{2}(B)\right\}$.

A short calculation shows that

$$
H_{G_{s_{1}, s_{2}}^{F}}(r) \sim s_{1}^{2 /(2 n+1)} s_{2}^{(2 n-2) /(2 n+1)} r^{1 /(2 n+1)} \text { as } r \downarrow 0 .
$$

In order to see that the bound in Corollary 5.5 can be better than Elsner's bound we define weighted shift matrices $A_{\epsilon}, B_{\epsilon} \in L\left(\mathbb{C}^{n}\right)$ by letting

$$
A_{\epsilon} e_{k}= \begin{cases}e_{2} & \text { if } k=1, \\
\epsilon e_{k+1} & \text { if } 1<k<n, \quad B_{\epsilon} e_{k}=\left\{\begin{array}{ll}
e_{2} & \text { if } k=1, \\
0 & \text { if } k=n,
\end{array} e_{k+1} \text { if } 1<k<n,\right. \\
\epsilon e_{1} & \text { if } k=n,\end{cases}
$$

where $\left(e_{k}\right)_{k=1}^{n}$ is an orthonormal basis of $\mathbb{C}^{n}$. Then we have

$$
\begin{gathered}
\left\|A_{\epsilon}-B_{\epsilon}\right\|=\epsilon, \\
s_{1}\left(A_{\epsilon}\right)=s_{1}\left(B_{\epsilon}\right)=1,
\end{gathered}
$$

and

$$
s_{2}\left(A_{\epsilon}\right) \leq s_{2}\left(B_{\epsilon}\right)=\epsilon,
$$

provided that $n>1$. For this family of matrices Elsner's bound gives

$$
H\left(\left\|A_{\epsilon}-B_{\epsilon}\right\|\right) \sim \epsilon^{1 / n} \quad \text { as } \epsilon \downarrow 0,
$$

which is worse than what we get from the bound in Corollary 5.5. namely

$$
H_{G_{1, \epsilon}^{F}}\left(\left\|A_{\epsilon}-B_{\epsilon}\right\|\right) \sim \epsilon^{(2 n-1) /(2 n+1)} \quad \text { as } \epsilon \downarrow 0 .
$$

In other words, our bound fares better then Elsner's for matrices which are small perturbations of matrices of rank one. Of course, this advantage is obtained by requiring more information about the matrices, namely upper bounds for the first and second singular values.

The previous discussion should have elucidated the following general principle at work here. Upper bounds for the singular values of $A$ and $B$ translate into upper bounds for the function $H_{F_{A, B}}$, which bounds the spectral variation of $A$ and $B$. Moreover, the faster the bounds for the singular values decay to zero, the slower $F_{A, B}$ will grow at infinity, and so, the faster $H_{F_{A, B}}$ will tend to zero at zero. 


\section{AcKnowledgements}

We are very grateful to an anonymous referee for bibliographic assistance. OFB would like to thank Henk Bruin at the University of Vienna for his kind hospitality from May until August 2014, during which period this article was finalised. AG expresses her gratitude to Wolfram Just for enlightening discussions during the preparation of this article.

\section{REFERENCES}

[1] O. F. BANDtLow, Estimates for norms of resolvents and an application to the perturbation of spectra, Math. Nachr. 267(1) (2004), 3-11.

[2] O. F. BAndtLow, Resolvent estimates for operators belonging to exponential classes, Integral Equations Operator Theory, 61 (2008), 21-43.

[3] O. F. Bandtlow, C.-H. Chu, Eigenvalue decay of operators on harmonic function spaces, Bull. Lond. Math. Soc. 41 (2009), 903-915.

[4] O. F. Bandtlow, O. Jenkinson, Explicit eigenvalue estimates for transfer operators acting on spaces of holomorphic functions, Adv. Math. 218(3) (2008), 902-925.

[5] R. Bhatia, Matrix Analysis, New York, Springer, 1997.

[6] Y. Chen, A. Nokrane, T. Ransford, Estimates for the spectrum near algebraic elements, Linear Algebra Appl. 308 (2000), 153-161.

[7] L. Elsner, An optimal bound for the spectral variation of two matrices, Linear Algebra Appl. 71 (1985), 77-80.

[8] M. I. GiL', Operator Functions and Localization of Spectra, Berlin, Springer, 2003.

[9] M. I. GIL', Norm estimates for resolvents of non-selfadjoint operators having HilbertSchmidt inverse ones, Math. Commun. 17 (2012), 599-611.

[10] M. I. GiL', Resolvents of operators inverse to Schatten-von Neumann ones, Ann. Univ. Ferrara (2013), DOI 10.1007/s11565-013-0200-1.

[11] I. Gohberg, S. Goldberg, M. A. Kanshoek, Classes of Linear Operators Vol. 1, Basel, Birkhäuser Verlag, 1990.

[12] P. Henrici, Bounds for iterates, inverses, spectral variation and fields of values of nonnormal matrices, Numer. Math. 4 (1962), 24-40.

[13] H. KöNIG, S. RiChTER, Eigenvalues of integral operators defined by analytic kernels, Math. Nachr. 119 (1984), 141-155.

[14] Y. LANGlois, T. RANSFORD, Counterexample to a conjecture of Elsner on the spectral variation of matrices, Linear Algebra Appl. 349 (2002), 193-195.

[15] J. D. Newburgh, The variation of spectra, Duke Math. J. 18 (1951), 165-175.

[16] A. Ostrowski, Über die Stetigkeit von charakteristischen Wurzeln in Abhängigkeit von den Matrizenelementen, Jber. Deutsch. Math. Verein 60 (1957), 40-42.

[17] A. Pokrzywa, On continuity of spectra in norm ideals, Linear Algebra Appl. 69 (1985), $121-130$.

[18] B. Simon, Notes on infinite determinants of Hilbert space operators, Adv. Math. 24 (1977), $244-273$.

Oscar F. Bandtlow, School of Mathematical Sciences, Queen Mary University of LONDON, LONDON E1 4NS, UK.

E-mail address: o.bandtlow@qmul.ac.uk

Ayşe Güven, School of Mathematical Sciences, Queen Mary University of London, LONDON E1 4NS, UK.

E-mail address: a.guven@qmul.ac.uk 